\title{
Comparative study of rational excavation methods for deep buried highway tunnel based on numerical simulation
}

\author{
Qiong Yan \\ Powerchina Roadbridge Group Co., LTD, Beijing, PRC, 100044 \\ ahoudinggui@126.com, byanqiongzz@163.com
}

Keywords: tunnel; finite difference; excavation method; stability of surrounding rock

Abstract. Based on the construction project of Guangshan No.1 tunnel of Jinhong high-speed. The finite difference software is utilized to study the tunnel excavation with 3 different construction methods, the surrounding rock stress, displacement and plastic zone are analyzed. The influence of different construction methods on the surrounding rock and support of tunnel is determined, some suggestions and the optimization scheme have been proposed, which could also be recommended for similar future projects.

\section{Introduction}

The highway tunnel in mountain area is affected by such factors as the shape of the mountain, the hilly area, the line of extension and the geological condition of the tunnel site. Moreover, the tunnel support structure is complex and the excavation and support are staggered, which make the stress change of surrounding rock and the transformation of supporting load become more complex, therefore, in the construction process, it is very important to control the safety and stability of the tunnel and optimize the supporting measures by adopting reasonable construction methods and construction procedures.

Different excavation methods have an impact on the stability of the stratum. Experts and scholars have studied it and obtained corresponding results ${ }^{[1]-[2]}$, however, the research on surrounding rock deformation and construction method of deep buried tunnel under complex geological conditions is not deep enough, the study of construction optimization needs to be strengthened, and the number of analog engineering examples can not be used for reference ${ }^{[3]-[7]}$.

\section{Engineering background}

Guangshan No. 1 tunnel is a separated tunnel of Jinhong high-speed, the distance between the left and right sides of the tunnel is $40 \mathrm{~m}$, and its total length is $733.56 \mathrm{~m}$, the tunnel entrance is shown in Fig.1.

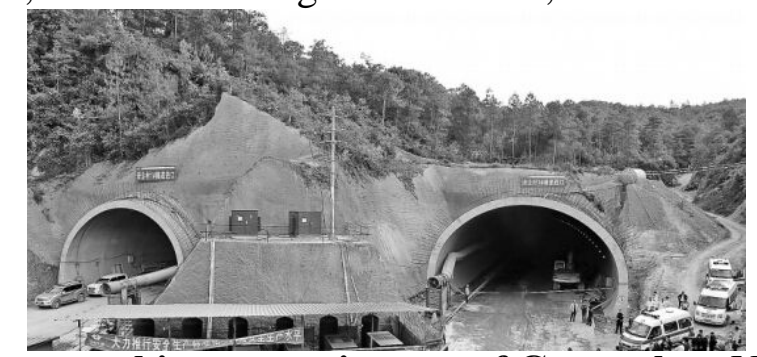

Fig.1 Highway tunnel in mountain area of Guangshan No.1 tunnel

\section{Design and excavation method}

The tunnel width is $14.50 \mathrm{~m}$, height is $5.0 \mathrm{~m}$, the composite lining with the initial support and the secondary lining is adopted. The anchor bolt, concrete, grille steel and profiled steel frame are used as the initial support, and the molded concrete is the secondary support lining. The bench cut method and core soil excavation method and CRD excavation method are compared and analyzed to study the characteristics of different excavation methods respectively.

The structure section is divided into three parts by bench cut method, which is divided into 3 working faces and excavated step by step. Bench cut method is a basic excavation method, which is 
adaptable, simple and flexible. The core soil excavation method divides the section into the annular arch part, the upper core and the lower part of the step. The core soil excavation method is flexible in excavation, large in working space and convenient for mechanical construction. CRD excavation method divides the tunnel into four small sections, and each section is closed for quick closure. Three kinds of excavation methods are shown in Fig.2.

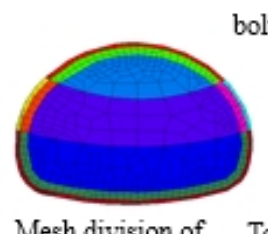

Mesh division of excavation section

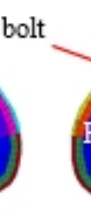

Top heading excavation and preliminary bracing

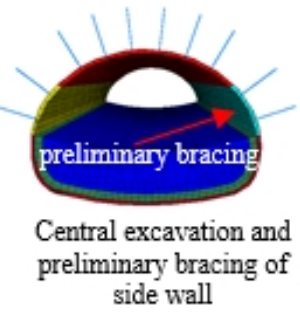
side wall

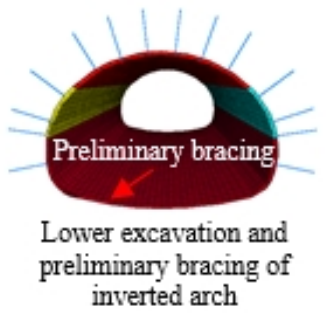

inverted arch

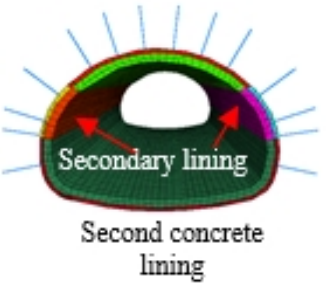

\section{a Bench cut method}

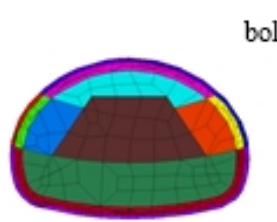

Mesh division of excavation section

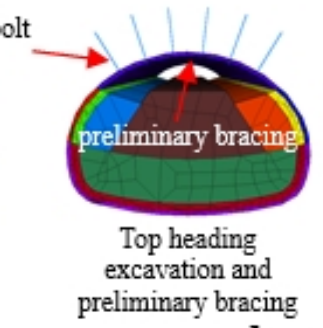

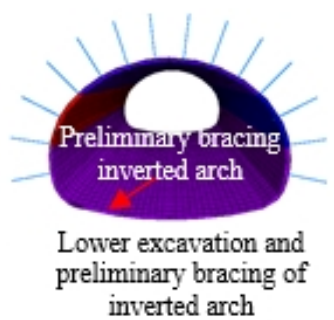

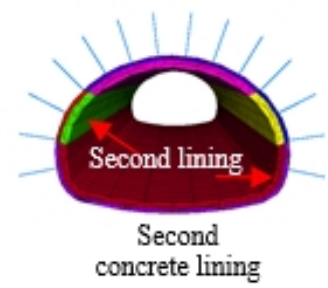

b core soil excavation method
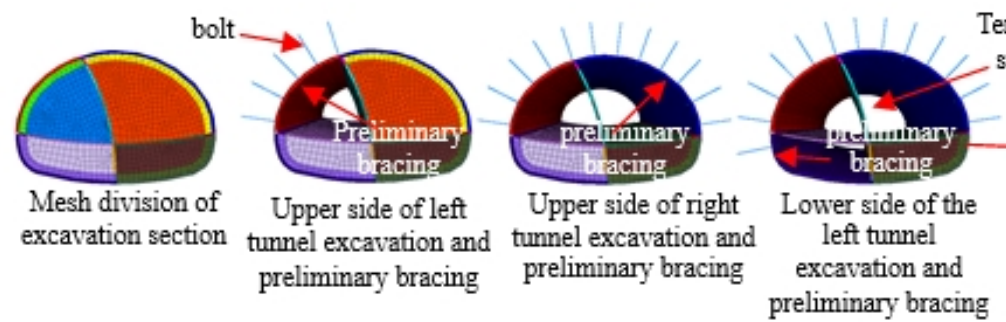

Temporary

support

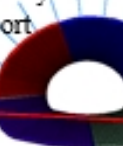

Lower side of the right tunnel excavation and preliminary bracing

\section{c CRD excavation method}

Fig.2 Construction sequence of excavation process with three methods

\section{calculation models and parameters}

The finite difference numerical software is used to simulate the dynamic analysis of different excavation modes. The finite difference model is shown in Fig.3.

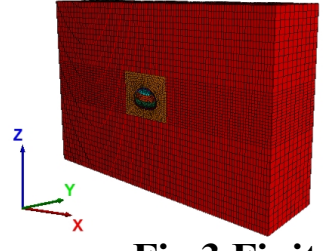

Fig.3 Finite difference model

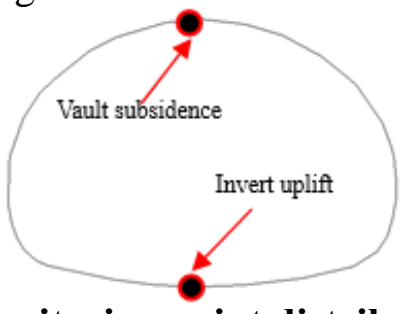

Fig.4 Monitoring point distribution

According to the geological survey report and design scheme in the design stage, the majority of the tunnel areas are in the slate rock stratum. Combining with the analogy method of similar engineering, the numerical calculation physical and mechanical parameters are given as shown in Table 1.

Table 1 Material parameter

\begin{tabular}{cccccc}
\hline Material & Density $/(\mathrm{kg} \cdot \mathrm{m}-3)$ & Cohesive strength $/ \mathrm{kPa}$ & Friction angle $/\left(^{\circ}\right)$ & Elastic modulus $(\mathrm{GPa})$ & Poisson ratio \\
\hline slate & 2500 & 850 & -37 & 0.55 & 0.38 \\
preliminary bracing & 2750 & - & - & 20 & 0.32 \\
Second lining & 2400 & - & - & 25 & 0.25 \\
Bolt & 7400 & & & 200 & 0.30 \\
\hline
\end{tabular}




\section{Calculation results analysis}

\section{calculation models and parameters}

We select the section ( $y=15 \mathrm{~m})$ as the target surface and set the monitoring point as shown in Fig.4. The arch dome settlement and the inverted arch uplift are monitored separately. The displacement curves of the arch dome and the inverted arch uplift by three kinds of excavation methods are shown in Fig.5 and Fig.6.

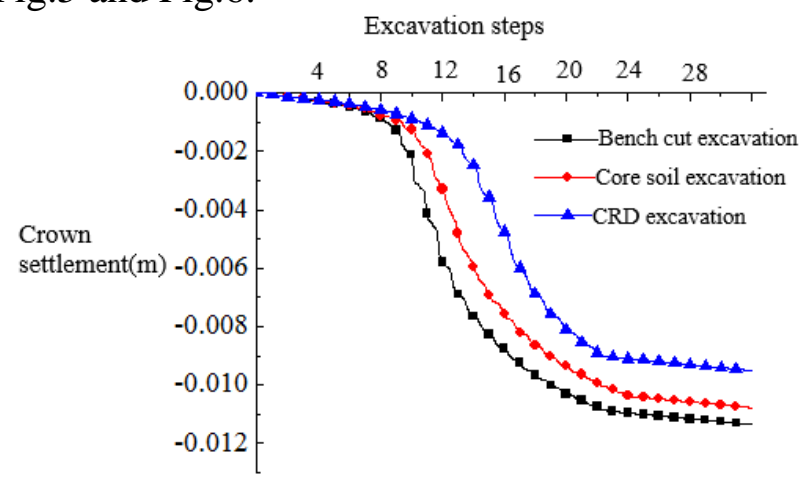

Fig.5 Vault subsidence varies

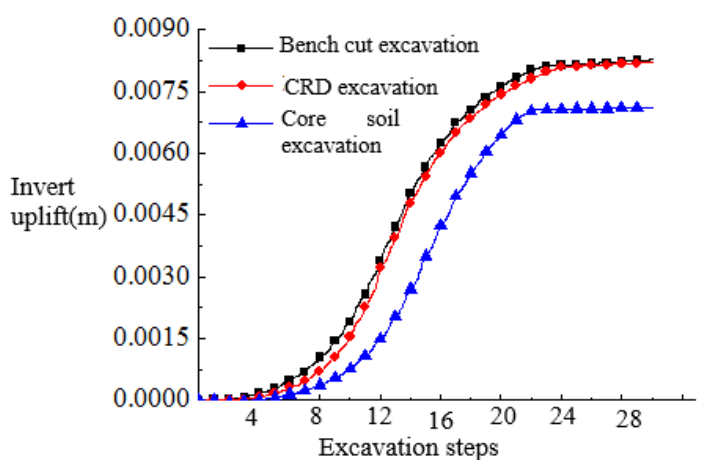

Fig.6 Variation curve of invert uplift

It can be seen that the CRD method has best effect on the deformation control of surrounding rock with deep high stress, and it can play the best controlling role in surrounding rock deformation. The core soil excavation method is similar to the bench cut method, but the addition of the core soils promote the control of the deformation of the tunnel surrounding rocks.

\section{Stress analysis of supporting structure}

The maximum principal stress distribution of the initial and second linings of the target section $(y=15 \mathrm{~m})$ after the stable excavation by three kinds of construction methods are shown in Fig.7, where the left side is the initial lining and the right side is the second lining.

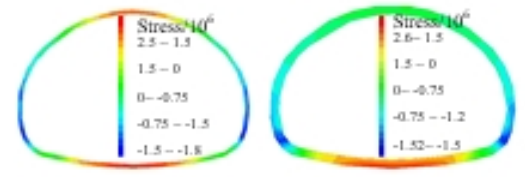

a Bench cut method

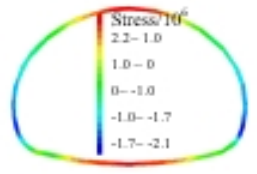

b core soil excavation method

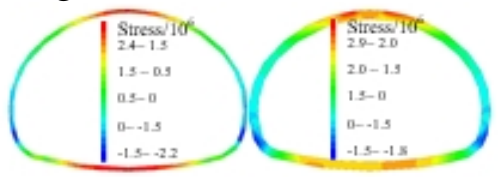

c CRD excavation method Fig.7 Distribution of maximum principal stress of retaining structure

The maximum stress of initial lining and lining is larger by using CRD method, the maximum stress is $2.9 \mathrm{MPa}$, which exceeds the strength of surrounding rock. At this time, the damage occurs easily in tunnel vault and arch bottom. This is due to the excavation procedures of CRD method are more than the other two methods, which make the wall rock masses disturbance greater. The maximum stress of the bench cut method is relatively small, which is due to its smaller excavation process, faster construction process, and less disturbance to the surrounding rocks. It also can be seen from the figures that the lining structures are prone to excessive settlement of arch crown, and the soils have the tendency of outward extrusion. The tunnel is mainly affected by the vertical stress, and the horizontal stress has a weak influence on the supporting structures.

\section{Analysis of plastic zone of surrounding rock}

After the tunnel surrounding rock is excavated to stability by using three kinds of construction methods, the distributions of surrounding rock plastic zone of the target section $(y=15 \mathrm{~m})$ are shown in Fig.8.

As shown in Fig.8, the range of plastic zone of surrounding rock is the largest by using the bench cut method and the smallest by using the CRD method. The plastic zone of the inverted arch area is the most widely distributed in the bench cut method excavation, and the vault also has the distribution of plastic area. There is plastic zone at the vault of the core soil excavation method, and the plastic 
zone at the inverted arch is significantly reduced. The plastic zones of vault and invert of CRD method are more stable than other methods.

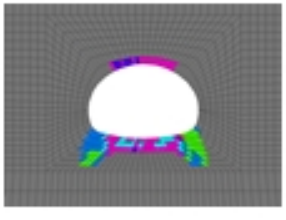

a Bench cut excavation

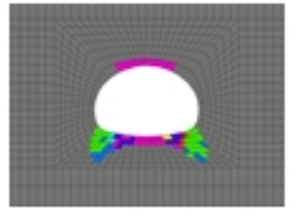

b core soil excavation

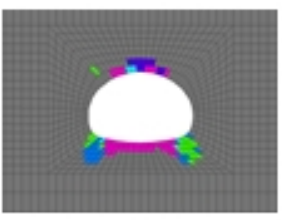

c CRD excavation

Fig.8 Plastic region distribution

The stress is concentrated at the inverted arch areas when the upper steps are excavated by using the bench cut method, which results in a large plastic deformation at the inverted arch. The core soil excavation method has controlled the development of plastic zone through the reservation of core soils, and the stability of surrounding rocks at tunnel inverted arch areas has been improved.

\section{Comparison and selection of construction schemes}

According to the numerical results of the bench cut method, core soil method and CRD method, combined with their applicability characteristics, the analysis results are shown in Table 2.

(1)CRD method has the best control effect compared to the bench cut method and the core soil method, and its initial and temporary supports have better control on the deformation of surrounding rocks. In the force of support structure, the CRD method is easy to form a larger principal stress. In the control of the plastic zone of the surrounding rocks, the core soil method is better to overcome the development of the plastic zone in the inverted arch areas of bench cut method. Additionally, the stability of surrounding rocks of the tunnel is well-controlled.

(2)The CRD method can play a great role of surrounding rocks control. However, its construction is more complex and temporary wall removal is more difficult, which are not conducive to the control of tunnel construction cost and time limit. The bench cut method and core soil method have enough construction space and fast construction speed, which increase the mechanical construction efficiency and reduces the construction cost compared to CRD method.

The core soil method is recommended in the excavation of Guangshan No.1 Deep-buried Tunnel, and the CRD method should be used to excavate the unstable rock strata. The engineering practice shows that the core soil excavation method is economical and feasible.

Table 2 Construction characteristics of different construction methods

\begin{tabular}{|c|c|c|c|}
\hline Construction method & Bench cut method & Core soil excavation method & CRD excavation method \\
\hline Difficulty & Low & Low & Higher \\
\hline Procedure & Less & Less & Complex \\
\hline Cost & Low & Low & Higher \\
\hline Construction period & Short & Short & Longer \\
\hline Tunnel face stability & Poor & Preferably & Better \\
\hline Deformation control & Commonly & Commonly & Preferably \\
\hline Applicability range & Ш Vgrade surrounding rock & Ш Vgrade surrounding rock & IV Vgrade surrounding rock \\
\hline
\end{tabular}

\section{Conclusions}

(1)According to the analysis of surrounding rock deformation and plastic zone distribution, the CRD excavation method is the best, followed by the core soil method and bench cut method. The plastic zone of the inverted arch is the most widely distributed in the bench cut method. In the deep-burial depth, the stability of inverted arch is one of the key factor for the stability of tunnel surrounding rocks.

(2)The reserved core soil has less influence on the soil without excavation by using the core soil method, while the support structure of the CRD method will bear the greater stress. Therefore, after 
comprehensive consideration, the reserved core soil method is more suitable for the tunnel excavation construction.

(3)In the construction of deep buried tunnel, the influence of vertical stress on supporting structure is greater than horizontal stress. In addition, the tunnel inverted arch is large upward, the corner parts have the destructive trend. So the initial lining and second lining should also strengthen the support of the inverted arch and corner parts.

(4)Through the stability study of numerical simulation and the comparison of scheme applicability, it is suggested that the core soil excavation method is adopted in the Guangshan No.1 Deep-buried Tunnel. Practices have proved that the excavation method is economical and effective.

\section{References}

[1] Eisenstein Z, Heinz H, Negro A. On three-dimensional ground response to tunneling[A]. ASCE. Geotech Ш, Tunneling in Soils and Rocks[C]. Atlanta: [s.n.], 1984.

[2] Huiling Duan, Lin Zhang. Comparative study of rational excavation methods for large span highway tunnels. 2009, 42(9):114-119.(in Chinese)

[3] Xiaolu Gu, Shimin Wand. Foundation[M].2ed ed. Beijing: Chinese Building Industry Press, 1991. (in Chinese)

[4] Xuefeng Cheng, Chenchen Yao, Jie Zhao. Simulating analysis for excavation methods of deep buried large-section highway tunnels. Highway engineering, 2015, 40(3): 152-156. (in Chinese)

[5] Yonghua Su, Xinliang He, Zhengdong Luo. Stability of surrounding rock mass based on strength reduction method[J]. Hydrogeological Engineering Geology, 2014, 41(1): 48-53. (in Chinese)

[6] Lan Cui, Wei Zhang, Junmin She, etal. Mechanical response of deep_buried tunnels through soft and cracked rock mass under different excavation methods[J], Journal of civil engineering and management, 2012, 29(4):36-40. (in Chinese)

[7] Chaoqiang Zhang, Le Tian, Bo Zhang. Analysis of deformation and failure mechanism of the high crustal stress soft rock tunnel during construction[J]. Highway, 2016,(1):228-234. (in Chinese) 